\title{
Lo studio dei microtoponimi del territorio di Alatri (Italia, FR) per una ricostruzione del quadro storico ed archeologico tra età romana e Medioevo
}

\section{Carmen Soria}

Scopo del presente contributo è l'analisi di alcuni toponimi e microtoponimi del territorio di Alatri (Italia, FR), al fine di individuare le presenze demiche nel comprensorio dal periodo romano a quello medievale, senza, tuttavia, soffermarsi sullo studio della centuriazione romana, per cui si rimanda al lavoro di Adriana Valchera $^{1}$. In questa sede, ci limiteremo a dimostrare come insieme ai toponimi romani coesistano quelli medievali in una zona di grande interesse storicoarcheologico. L'area in esame, infatti, la Valle del fiume Sacco, tra il Lazio e la Campania, nella Ciociaria orientale ${ }^{2}$, facente capo a Frosinone (Lazio, a Sud-est di Roma), e delimitata a Nord dai monti Ernici e dai monti Ausoni a Sud, è da sempre stata interessata dal percorso dell'antica Via Latina che collegava Roma a Capua ${ }^{3}$. I centri più importanti di questa area, oltre a Frosinone, capoluogo, sono Alatri, a Nordovest e Collepardo a Nord, Frosinone a Sud e Veroli ad Est. Innanzitutto, ci preme sottolineare che, ad una prima analisi, su un totale di 92 toponimi e microtoponimi esaminati, ben 32 sono riferibili ad infrastrutture, viabilità e ripartizione territoriale (centuriazione, prediali, fitotoponimi), di epoca romana e medievale; seguono gli agiotoponimi, $18^{4}$.

Partendo dal gruppo più considerevole, quello dei toponimi riferibili alla viabilità e alle infrastrutture, nonchè alla ripartizione territoriale, 9 sono riconducibili alla centuriazione romana: basti considerare Arillette, possibile derivato di arger, argine, nel senso di linea di confine o di Carano, riferibile a quadra, campo, appezzamento di terra di forma quadrata, entrambi di matrice latina ${ }^{5}$. Altro microtoponimo interessante, visibilmente legato alle infrastrutture, è Fosso del Bagno, composto da fossum, corso d'acqua con avallamento e da balneum, acqua termale, indicativo, probabilmente, di una struttura termale romana, molto frequente nella toponomastica ${ }^{6}$; relativamente al primo termine, Fosso, dobbiamo dire che le fossae, nel linguaggio gromatico romano, facevano parte di quelle opere di canalizzazione realizzate per drenare I campi ${ }^{7}$. Ancora, sempre appartenente alla stessa classe, è Laguccio, diminutivo da lacus, piccolo fossato, avvallamento, spesso riferito ad argine romano di contenimento in terreno paludoso, nel mezzo della riparttizione agraria del luogo ${ }^{8}$. I microtoponimi Quarti di Tecchiena, Quarticciolo e Quartiere

1 VALCHERA 2000, 50-53.

2 AA.VV, Lazio : non compresa Roma e dintorni, Guida d'Italia. Milano 1981, 556. Si rende necessario precisare che, spesso, siamo in presenza di tiponimi “ fossili “, ovvero di nomi le cui realtà geomorfologiche o demiche che li connotavano sono così profondamente mutate, da non essere più verificabili fisicamente e per questo motivo ancora più preziosi nella ricostruzione topografica.

3 Siamo nel 328 a. C.: questa strada, che collega l'attuale Lazio alla Campania, segue il tracciato naturale della Valle del Sacco e del fiume Liri. Basso 2007, 15.

4 Su di un totale di 92 toponimi analizzati, ne sono stati scelti 62 per una trattazione analitica, secondo un criterio di maggiore rappresentatività. I geonomastici, che si riferiscono alla morfologia del terreno, pur essendo, per quantità, al secondo posto, 23, sono ripresi e trattati in un secondo momento.

5 DizTop. 2006, 45, 163.

6 DizTop. 2006, 332. Si veda, inoltre, il contributo di Uggeri, in particolare il paragrafo sulle classi semantiche (UGGERI 2000, 119-132).

7 FILIPPI 2003, 148 in AA. VV., Misurare la terra: centuriazione e coloni nel mondo romano, Modena 2003.

8 PELLEGRINI 1990, 384-385, 400. 
Vicinale, ager qui ad quartam partem tenetur, sono riferiti a quartus, quarto, distanza stradale romana, relativa alla rete stradale e costituiscono una voce molto diffusa nel territorio italiano ${ }^{9}$. Proprio quest ultimo toponimo, può essere assimilato alle viae vicinales, ossia a quelle vie private che devertuntur in agris et saepe ipsae ad alteras publicas perveniunt ${ }^{10}$. Via Latina, si riferisce chiaramente alla viabilità romana di epoca repubblicana, aperta a ridosso della definitiva conquista del Latium con la sottomissione degli Equi e dei Volsci ${ }^{11}$, così come Vicero, è da ricondursi a vicus, ad indicare un tipo di abitato, di fondovalle, molto probabilmente, ripreso in età altomedievale col significato di gruppo di case, quartiere o semplicemente abitato sparso $^{12}$. Allo stesso modo, Preturo, da praetorium, dimora, palazzo del pretore, poi, casa signorile di campagna o acquartieramento, sempre di derivazione latina ${ }^{13}$.

Sempre riconducibili alle forme insediative, ma di chiara origine medievale, riscontriamo, nello stesso gruppo: Abbadia, dal latino tardo abbatia, da abbas, abate, indica località sorte intorno ad un'abbazia, soprattutto antica, Castello, dal latino castellum, diminutivo di castrum, fortezza, campo fortificato, sebbene, spesso, più che ad una struttura alluda, per traslato, nelle zone montuose a roccia a picco di difficile accesso. Ancora: Magione, gallicismo, maison (XI), derivato dal latino mansio, famiglia (monacale) da cui convento, dimora dove si pernotta, quindi casa con podere $^{14}$, e Osteria di Alatri, dal latino medievale hostaria, da hospitem, latino volgare (sec. XIII) $)^{15}$, luogo deputato all'accoglienza dell'ospite; infine, Case Paolone, dal latino casa, casalis, con sottinteso fundus, case abitate di servi o coloni, gruppo di case, soprattutto nel Medioevo, aggregato di case rurali (a cui segue il nome moderno della zona/del proprietario, così come appare nelle particelle catastali). Lo stesso, dicasi per Villa Magna, composto di Villa, agglomerato urbano rurale, paese o fattoria nel latino medievale, più la specifica magnus, grande, chiaramente riferito ad un agglomerato rurale di notevole estensione ${ }^{16}$. Riconducibile all'uso del suolo è, invece, Cellerano, da cella, molto frequente, spesso nel senso di grotta, e per esteso ma anche deposito di derrate, che trova numerosi riscontri nella Penisola soprattutto nel secolo XII: Cellem, Cellas ovvero, luoghi di raccolta dell'annona militare, il più delle volte vicino ad un castrum e ad una viabilità antica, spesso preesistente, romana ${ }^{17}$.

Riguardo ai termini relativi alla gromatica medievale, quasi la maggior parte sono fitotoponimi come da tradizione, infatti, il più delle volte, su un dato territorio permangono i segni linguistici del diritto in uso presso le popolazioni di origine germanica, soprattutto longobarda, di delimitare i confini mediante segni naturali del territorio, soprattutto alberi e piante. Infatti riscontriamo Quercia d'Orlando, dal fitonimo quercus, quercia (più l'antroponimo Orlando), a delimitazione di un'area o di una viabilità secondaria, secondo l'uso gromatico medievale ${ }^{18}$. Castagneto, da castanea, castagna, con terminazione collettiva in etum-, luogo dove abbondano castagni, molto diffuso nella documentazione medievale (dalla silva castanearum alla

9 PELLEgRINI 1990, 197-198, 384-393. Ancora, si rimanda a UGGERI 2000, 119-132.

10 ECK 1999, 76, nota 271. Si veda anche il contributo di Capogrossi Colognesi (Capogrossi Colognesi 2003, 28-32 in AA. VV., Misurare la terra: centuriazione e coloni nel mondo romano, Modena 2003).

11 Si veda la nota 2.

12 PELLEGRINI 1990, 235; PELLEGRINI 2006, 823.

13 DizTop. 2006, 615.

14 D.E.L.I., III, 700.

15 DizTop. 2006, 3, 197-198. PELlegrini 1990, 221; D.E.L.I., IV, 852.

16 DizTop. 2006, 827, 830.

17 Dal quale deriva il sostentivo Cellarius: cui potus et escae cura est, qui cellae vinariae, in DizTop. 2006, 219-220.

18 PELLEGRINI 1990, 348-349; UGGERI 2000, 125, WERMÜLLER 1990, 461-476. 
terra sementaricia cum arboribus castanearum, nel Lazio dei secoli XI-XII è il paesaggio più diffuso ${ }^{19}$ ) così come Porpuro, probabilmente dal fitonimo populus, pioppo, con suffisso collettivo in etum-, luogo di pioppi, o, ancora, Melegranate, dal fitonimo malum granatum, frutto del melograno ${ }^{20}$. Sempre tra i fitotoponimi, abbiamo Vignola, da vinea vigna, Fontana Sambuco, composto da fons, fonte più sambucus, albero del sambuco ${ }^{21}$. Ancora: Fraschette, da frascarium, boschetto di cespugli, Frittola da fractu, bosco reciso/boscaglia di difesa, Gaudo, post latino, dal tedesco wald, bosco, chiramente relativi all'uso diversificato delle aree silvo-pastorali, già a partire dal secolo VIII' ${ }^{22}$.

Un discorso a parte, merita Tecchiena: il toponimo, infatti, che già compare nella documentazione locale alla fine del secolo $\mathrm{IX}^{23}$, Teclena, viene riferito a ticlus, segno di confine o rupe, o ancora: techna, imboscata, astuzia, a designare un luogo dove si tessono imboscate, data la natura boscosa. In realtà, l'interpretazione più plausibile sembra la prima, ovvero segno di confine: teclaturas/teclatus incisione in un albero come segno di confine/ che reca inciso un segno, tipico del diritto consuetudinario germanico, già presente nell'Editto di Rotari: ticlatura aut snaida ${ }^{24}$.

Un gruppo interessante, molto significativo, è quello composto dai prediali, che attestano la presenza degli antichi proprietari dei fondi, praedia, sono 6 e sono tutti caratterizzati dalla terminazione, tipica, in anus-: Cassiano, prediale dal personale latino Cassius $^{25}$, Coste Rasciano, costa, fianco del colle/monte seguito dal prediale Rantius. Intignano, prediale latino da Leninius ${ }^{26}$, Magliano, prediale dall'antroponimo latino Mallius, con terminaz aggettivale in anus, ad indicare proprietà fondiaria; Maranillo, dal personale latino Marius, con suffisso diminutivo da terminaz aggettivale in anus, ad indicare proprietà fondiaria, infine, Riano, prediale da un antroponimo latino, Arrius $^{27}$.

Altro gruppo altrettanto importante è quello degli antroponimi, tutti di origine latina, tranne Collepardo: composto dal geonimo collis più l'antroponimo germanico, longobardo Pardo, attestato dal secolo VIII; Basciano, derivato da un personale latino, Bassius, con suffisso anus, ${ }^{28}$. Ancora: Pacciano, da Paccius, antroponimo, Vado Bazzano, vadum, guado, acqua bassa, ed anche valico, passaggio più personale latino, Badius, a designare proprietà fondiaria; Varignano, antroponimo latino, da Varinius ${ }^{29}$. Infine, Colletraiano e Fontana ${ }^{30}$ Sistiliana, si riferiscono, chiaramente, alle vicende storiche del territorio: il primo è legato all'imperatore romano (fine I-inizio II d.C., al quale si deve la massima espansione territoriale dell' impero e la massima espressione dell'utilizzo dell'agro centuriato) ${ }^{31}$, il secondo, a Sisto I, papa e coopatrono di Alatri

19 TOUBERT 1997, 79.

20 DizTop. 2006, 187, 603-604, 458.

21 Pellegrini 1990, 176, 356-357, 181, 351.

22 TOUBERT 1997, 189.

23 BOEZI 2004, 20.

24 Insieme a staffilus e snaida fa parte di termini longobardi indicanti I segni di ripartizione del territorio, di confine; per una trattazione esaustiva, si veda: PRINCI BRACCINI 1999, 213.

25 DizTop. 2006, 186.

26 PELLEGRINI 1990, 179, 321, 315.

27 DizTop. 2006, 436, 443, 631.

28 DizTop. 2006 76, 257.

29 Pellegrini 1990, 318, 205, 324; DizTop. 2006, 79. Si tratta di antroponimi relativi a cognomina latini.

30 Fontana, già attestato nel secolo XIII: costruzione destinata a raccogliere e distribuire l'acqua di una sorgente o di una condotta; D.E.L.I, II, 1980, 448.

31 Celuzza 2003, 201-203; Pellegrini 1990, 176. 
(132-142), le cui spoglie riposano ivi ${ }^{32}$. Ancora una volta, dunque, i residui toponimici sono di fondamentale importanza per ricostruire le dinamiche storicoinsediative del paesaggio.

Trattando di toponimi, ovviamente, dobbiamo necessariamente considerare gli agiotoponimi, utili sia per l'identificazione dei culti e dei relativi tituli, sia perchè si riesce, spesso, tramite l'origine di essi, a risalire anche alle popolazioni sul territorio presso cui erano maggiormente in uso in quel dato momento storico. Ad esempio, San Manno e San Pancrazio: del primo, possiamo solo ipotizzare si tratti di un nome di origine marcomanna (Mann- $)^{33}$; per il secondo, sappiamo che è di origine siria/frigia, martire nel 304 e il cui culto è diffuso massimamente sin dal secolo VI, nel periodo della guerra greco-gotica (535-553) ${ }^{34}$. Gli altri agionimi sono: San Mattia, di I secolo, San Valentino e Santa Colomba, entrambi di III, Santa Caterina e Santo Emidio, entrambi di $\mathrm{IV}^{35}$. Tra gli agiotoponimi, ovvero, i toponimi composti derivati da nomi di santi, abbiamo, risalenti al secolo I: Valle San Matteo e Fontana Santo Stefano ${ }^{36}$. Infine, Molto interessanti, inoltre, sono: Maddalena, Madonna del Pianto e Madonna delle Grazie; questi tre toponimi, normalmente, sono spesso riferiti a edicole/chiesette rurali, il più delle volte poste lungo percorsi tratturali ${ }^{37}$. Infine, Monte San Marino, e Montesantangelo: il primo risalente al secolo VII, il secondo, invece, è molto diffuso nel XII, lungo gli itinerari rupestri, meta di pellegrinaggi ${ }^{38}$.

Passando al gruppo dei geonomastici, toponimi legati alla conformazione del terreno, la maggior parte presentano una base collis, vallis, campus-ulus o mons e si riferiscono, chiaramente, a sommità di piccole o grandi dimensioni o ad appezzamenti di terra più la specifica (p.e.: Montelarena) ${ }^{39}$. I geonimi Pezza e Pezzelle, da petia, pezzo di terra, sono utili per darci l'idea dell'assetto rurale della zona, infatti, ne descrivono la tipica conformazione per "pezze", di forma irregolare, non sempre regolari o allineate come nella centuriazione romana; basti considerare la documentazione in cui appaiono nelle forme peciola, petja de terra ${ }^{40}$. Altri, sebbene sempre legati alla conformazione del terreno, sembrano anche indicare un uso del suolo specifico, come nel caso di Canale, da canalis, condotto per l'acqua, allude ad un sistema di irrigazione e Canarolo, sua variante ${ }^{41}$ o come nel caso di Capranica, da capra, luogo frequentato o destinato all'allevamento dei caprini ${ }^{42}$. Infine, benchè legati più espressamente ad un'attività precisa, abbiamo: Mole Bisleti e Mole Santa Maria (agiotoponimo), derivanti da Mola, macina, ad indicare una zona di attività di macinazione del grano, per ottenere la farina ${ }^{43}$; ancora una volta, lo studio della micro

32 Pellegrini 1990, 181; CAPPELli 1998, 185.

33 Forse si tratta di un diminutivo per Ermanno. FrANCOVICH ONESTI 2000, 207; BRACCINI 19981999, 194.

34 CECCHELli 1972, 5.

35 Pellegrini 1990, 398-403. CAPpelli 1998, 176, 187, 162, 160, 164.

36 PELLEGRINI 1990, 398-403. CAPPELLI 1998, 176, 185-186.

37 DizTop. 2006, 434-435. CAPPELLi 1998, 175, 147-148. Si tratta di culti tardo medievali, la cui diffusione massima si ha nei secoli XVI-XVII.

38 CAPPELLI 1998, 175, 158. UGGERI 2000, 128.

39 Pellegrini 1990, 176, 206, 172-173, 192, 169. Qui la specificazione deriva da arena, sabbia ed alluderà alla composizione del rilievo.

40 DizTop. 2006, 575. BRACCINI 1999, 209.

41 Pellegrini 1990, 173. Si veda anche il contributo sulla terminologia agraria, sempre dello stesso autore: PELLEGRINI 1966, 615-616.

42 DizTop. 2006, 159-160. In questo caso, si tratta, nello specifico, di uno zootoponimo perchè prende origine dall'animale che caratterizza/caratterizzava il paesaggio e la cui presenza si lega/legava ad attività pastorali e commerciali.

43 DizTop. 2006, 471. 
toponomastica può fornirci l'idea di quello che poteva essere il paesaggio agrario e le attività ad esso legate. Resta, invece, fuori da una possibile classificazione, almeno per il momento, il toponimo Alatri: esso, infatti, si presenta nella forma di genitivo locativo di Áśćııov (Strabone, III, 10) ${ }^{44}$, preromano, centro degli Ernici, poi Municipium nel periodo della Guerra Sociale ${ }^{45}$.

Prima di concludere, tornando al sopracitato lavoro di Adriana Valchera ${ }^{46}$, alcuni dei toponimi fin qui esaminati, risultano concentrarsi nell'area interessata dai resti della centuriazione romana: Tecchiena, Colle Cattrino, Cavariccio, Campone, Osteria di Alatri, Laguccio, Arillette, Quercia di Orlando, Frittola. Ad una attenta analisi, questi microtoponimi si dividono abbastanza equamente tra voci legate all'uso di marcare il territorio secondo elementi naturali, tipici del diritto germanico altomedievale, come abbiamo già visto, soprattutto evidenti per Tecchiena, Quercia di Orlando, Frittola, Campone. Si tratta, infatti, di fitotoponimi, agiotoponimi (per ovvie ragioni cronologiche) e di alcuni toponimi legati alle forme insediative sul territorio di origine tardo latina/altomedievale: Abbadia, Magione, Mole, Castello. Di contro, dobbiamo aggiungere che gli altri, Colle Cattrino, Cavariccio, Laguccio e Arillette insieme ai prediali, ai geonomastici e quelli riferiti alle infrastrutture e alla centuriazione, sono di origine romana: $i$ coltivatori anche nel medioevo continuano ad essere $i$ romani che per secoli perpetuano più o meno - salvo rare eccezioni - le identiche tecniche di lavorazione agricola e quasi identici nomi ${ }^{47}$.

Da quanto esaminato, si evince come, per questa area, parallelamente alla continuazione di microtoponimi della gromatica latina, vadano a sovrapporsi termini toponomastici secondo due modalità: nella prima si sovrappongono all'origine romana, cambiandone, però la destinazione d'uso, basti il caso di Villamagna, per il quale, pur derivando da un termine latino, villa, nel significato di casa di campagna, fattoria, viene a a designare nei documenti altomedievali di IX un agglomerato urbano rurale, un paese (a volte anche una fattoria), tipico dell'abitato sparso, dove la specifica magna, la distingue da altre villae nel territorio ${ }^{48}$. Nel secondo caso, invece, per esempio per Abbadia, è evidente che siamo in presenza di una voce nata ex novo, legata, storicamente, alla presenza sul territorio di una comunità monastica. A tal riguardo, esemplare è il lavoro sulla microtoponomastica storica del Lazio meridionale di Paul Toubert: lo studioso, infatti, analizzando le carte relative ai toponimi della "riconquista agraria" osserva come sono grosso modo ripartiti in parti uguali i toponimi romani di costruzioni antiche e quelli medievali ${ }^{49}$.

44 BIRASCHI 1988, 148.

45 DizTop. 2006, 16. Ritarossi 1999, 8.

46 VALCHERA 2006, 50-54, in particolare, si veda la fig. 65.

47 PELLEGRINI 1966, 659-660.

48 TOUBERT 1997, 102.

49 TOUBERT 1997, 66, nota 67. 
Frankfurter elektronische Rundschau zur Altertumskunde 11 (2010)

\section{BIBLIOGRAFIA E ABBREVIAZIONI}

\section{AA.VV. 2006}

Dizionario di Toponomastica, Milano 2006.

AA.VV. 1981

AA.VV., Lazio : non compresa Roma e dintorni, Guida d'Italia. Milano 1981.

Basso 2007

Basso P., Strade romane: storia e archeologia, Roma 2007.

Biraschi 1988

Biraschi A. M., Strabone. Geografia: l'Italia, Libri V-VI, Milano 1988.

Boezi 2004

Boezi G., Storia di una torre antica, Alatri 2004.

Capogrossi Colognesi 2003

Capogrossi Colognesi L., Servitù di passaggio e organizzazione del territorio romano nella media e tarda età repubblicana in AA. VV., Misurare la terra: centuriazione e coloni nel mondo romano, Modena 2003, 28-32.

Cappelli 1998

Cappelli A., Cronologia, cronografia e calendario perpetuo, Milano 1998.

Cecchelli 1972

Cecchelli M., S. Pancrazio, Roma 1972.

Celuzza 2003

Celuzza M., La centuriazione nell'impero romano in Misurare la terra: centuriazione e coloni nel mondo romano, Modena 2003, 201-203.

Ceraudo 2006

Ceraudo G., Le collezioni dell'Aerofototeca Nazionale per la conoscenza del territorio: la provincia di Frosinone, Frosinone 2006, 1-119.

Chiappinelli 2002

Chiappinelli L., Lessico Idronomastico dell'Abruzzo e del Molise, in Quaderni di AISN, Nuova Serie 7, Napoli 2002, 1-149.

D.E.L.I. (= Dizionario Etimologico della Lingua Italiana) 1979-1988

D.E.L.I., a cura di M. Cortellazzo - P. Zolli, I-V, Bologna 1979-1988.

DizTop (= Dizionario di Toponomastica) 2006

Dizionario di Toponomastica, a cura di Gasca Queirazza G., Marcato C., Pellegrini G. B., Milano 2006.

Eck 1999

Eck W., L'Italia nell'impero romano. Stato e amministrazione nell'epoca imperiale, Bari 1999. 
Frankfurter elektronische Rundschau zur Altertumskunde 11 (2010)

Filippi 2003

Filippi M. R., Le procedure: il paesaggio centuriato in Misurare la terra: centuriazione e coloni nel mondo romano, Modena 2003, 147-150.

Francovich Onesti 2000

Francovich Onesti N., Vestigia longobarde in Italia (658-774), Lessico e antroponimia, Roma 2000.

Pellegrini 1966

Pellegrini G. B., Terminologia agraria medioevale in Italia in Agricoltura e mondo rurale in Occidente nell'alto medioevo, Atti della Settimana di Spoleto (Spoleto 22-28 aprile 1965), Spoleto 1966, 605-661.

Pellegrini 1990

Pellegrini G. B., Toponomastica italiana, Milano 1990.

Princi Braccini 1998-1999

Princi Braccini G., Germanismi editi e inediti nel Codice Diplomatico Longobardo: anticipi da uno spoglio integrale e commentato di fonti latine in vista di un tesoro longobardo in Quaderni del Dipartimento di Linguistica - Università di Firenze 9, Firenze 1998/99, 191-240.

Ritarossi 1999

Ritarossi M., Aletrium, Alatri 1999.

Toubert 1997

Toubert P., Dalla terra ai castelli. Paesaggio, agricoltura e poteri nell'Italia medievale, Torino 1997.

Uggeri 2000

Uggeri G., Il contributo della toponomastica alla ricerca topografica, in AA. VV., La topografia antica, Bologna 2000, 119-132.

Valchera 2006

Valchera A., Frosinone e la Valle del Sacco, in Le collezioni dell'Aerofototeca Nazionale per la conoscenza del territorio: la provincia di Frosinone, Frosinone 2006, 50-53.

Wermüller 1990

Wermüller D., Gli alberi come segno di confine e luogo di giudizio nel diritto germanico medievale, in L'ambiente vegetale nell' alto medioevo (Spoleto 30 marzo-19 aprile 1989), Spoleto 1990, 461- 476.

Carmen Soria DSC

Università degli Studi “G. d'Annunzio”

Chieti (Italy)

E-Mail: carmensoria73@gmail.com 